\title{
А.В. Шатравин
}

\section{ОЦЕНКА ЭФФЕКТИВНОСТИ ПОДВОДНОЙ АКУСТИЧЕСКОЙ СВЯЗИ НА АРКТИЧЕСКОМ ШЕЛЬФЕ НА ОСНОВЕ КЛИМАТИЧЕСКИХ И ИЗМЕРЕННЫХ ПРОФИЛЕЙ СКОРОСТИ ЗВУКА}

\author{
Институт океанологии им. П.П. Ширшова РАН \\ Россия, 117997, Москва, Нахимовский проспект, 36, \\ Тел.: 8(499)124-59-96, E-mail: ashatravin@ocean.ru
}

\begin{abstract}
Представлены результаты численного моделирования подводной акустической связи на основе климатических и измеренных вертикальный профилей скорости звука на мелководном шельфе российской Арктики. Показано, что уровень ошибки декодирования для измеренных профилей может отличаться от прогнозируемого на основе климатических данных более, чем на 6 дБ. Доля битовых ошибок декодирования для измеренных профилей выше в среднем на 0.031 .
\end{abstract}

Ключевые слова: подводная акустическая связь, арктический шельф, климатические профили, лучевая модель

Подводная акустическая связь (ПАС) во многих случаях оказывается оптимальным способом передачи цифровой информации между находящимися под водой объектами, например, автономными аппаратами и океанологическими измерительными комплексами. Существует множество методов ПАС. Выбор протоколов, алгоритмов и их параметров для каждой конкретной задачи зависит от предъявляемых требований к скорости и надежности связи. При этом условия распространения звука в океане так сильно зависят от меняющихся во времени условий среды, что даже для фиксированных источников и приемников один и тот же подход может безошибочно работать в один день и оказаться совершенно бесполезным в другой [1]. Правильно выбрать методы кодирования сигнала, частотный диапазон, скорость передачи информации, мощность источника и т.д. может помочь моделирование связи на основе доступных данных о характерных для региона работ вертикальных профилях скорости звука. Одним из общедоступных источников такого рода данных является климатический атлас World Ocean Atlas (WOA) [2]. В представленной работе сравниваются результаты моделирования ПАС 
для климатических и реально зарегистрированных на мелководном шельфе Арктики профилей скорости звука, и таким образом оценивается снизу ошибка прогнозирования эффективности связи, обусловленная обобщенным характером климатических данных.

Набор измеренных профилей температуры и солености был сформирован из представленных в базе World Ocean Database (WOD) [3] профилей с географическими координатами от $70^{\circ}$ до $80^{\circ}$ с.ш. и от $60^{\circ}$ до $180^{\circ}$ в.д., глубиной от 30 м до 350 м и удовлетворяющих некоторым простым критериям качества измерений. Всего было отобрано 237 профилей, преимущественно в Карском море. Для каждого из измеренных профилей был построен соответствующий климатический профиль путем интерполяции по глубине профиля из атласа WOA для тех же координат и месяца измерений. Скорость звука рассчитывалась по формуле Дель-Гроссо. Географические координаты и распределение глубин и месяцев измерений для составленного набора профилей представлены на рис. 1.
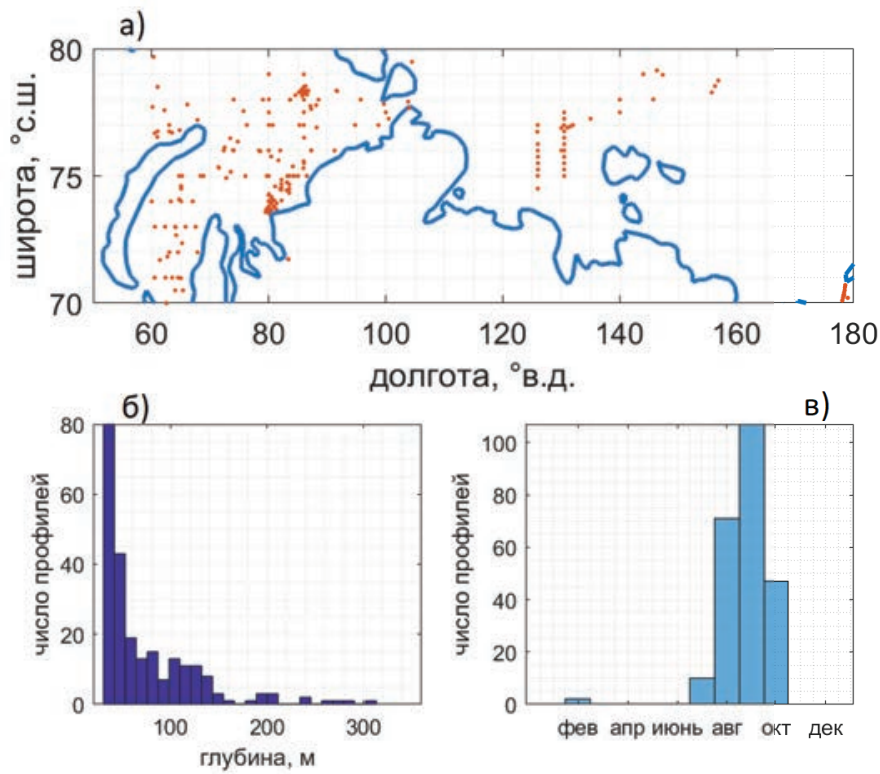

Рис. 1. Географические координаты профилей (а), гистограммы глубин (б) и месяцев измерений (в).

Моделирование распространения сигналов связи выполнялось с использованием лучевой программы Bellhop [4]. Принятый сигнал форми- 
ровался методом когерентного суммирования по приходам собственных лучей. Среда предполагалась плоскослоистой и постоянной в течение передачи сигнала связи. Излученный сигнал представлял собой преамбулу в виде $м$-последовательности и следующую за ней случайную информационную последовательность, модулирующие методом двоичной фазовой манипуляции несущий сигнал на частоте 10 кГц. Скорость передачи информации составляла 2000 бит/с (5 периодов несущей на символ). Расстояние между придонными (1 м от дна) одиночными приемником и источником составляло 1 км. Для большинства профилей длина трассы составила более 10 глубин волновода. Скорость звука в дне полагалась равной 1400 или 1600 м/с (для каждого профиля расчеты проводились при обоих значениях), коэффициент затухания в дне 0.5 дБ на длину волны, плотность дна -1.8 г $/ \mathrm{cm}^{3}$.

Эффективность связи оценивалась по уровню среднеквадратичной ошибки мягкого решения декодирования с применением эквалайзера с обратной связью MMSE-DFE [5]. Оценка импульсного отклика для подбора параметров эквалайзера и построения коэффициентов фильтров прямой и обратной связи осуществлялась по кросс-корреляционной функции принятой и излученной преамбулы. В отсутствие аддитивного шума, получаемая таким образом оценка эффективности связи соответствует уровню остаточной межсимвольной интерференции после применения эквалайзера. Результаты декодирования представлены на рис. 2. Стандартное отклонение разности уровней ошибки декодирования для измеренных и климатических профилей составило 6.2 дБ при среднем 1.3 дБ. Это означает, что в реальных условиях граничные значения мощности аддитивного фонового шума, позволяющие достигать приемлемого уровня битовых ошибок декодирования, в зависимости от гидрофизических условий, могут в разы отличаться от спрогнозированных на основе климатических данных. Таким образом, при планировании ПАС на основе анализа климатических данных, необходимо закладывать многократный запас по мощности излучения.

В качестве примера была рассмотрена дополнительная помеха в виде аддитивного белого гауссовского шума (АБГШ). Принятые сигналы связи суммировались с реализациями АБГШ одинакового для всех профилей уровня и декодировались с применением эквалайзера MMSEDFE. Среди пар климатических профилей и значений скорости звука в дне были выбраны те, для которых доля битовых ошибок декодирования (BER) составила менее 0.1 (таких моделей среды оказалось 303), и проведено сравнение полученных для них значений BER со значениями BER для измеренных профилей (рис. 3). 

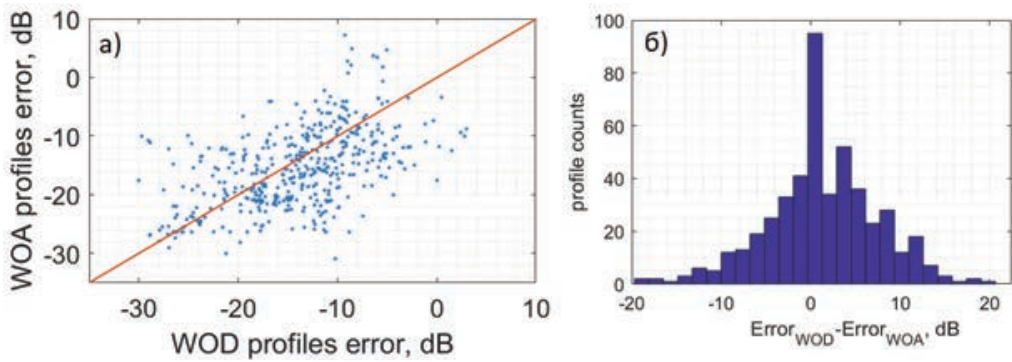

Рис. 2. а) уровень ошибки декодирования для измеренных профилей (по горизонтальной оси) и климатических профилей (по вертикальной оси); б) гистограмма разности уровня ошибки декодирования (положительные значения означают более высокий уровень ошибки для измеренного профиля, чем для климатического).
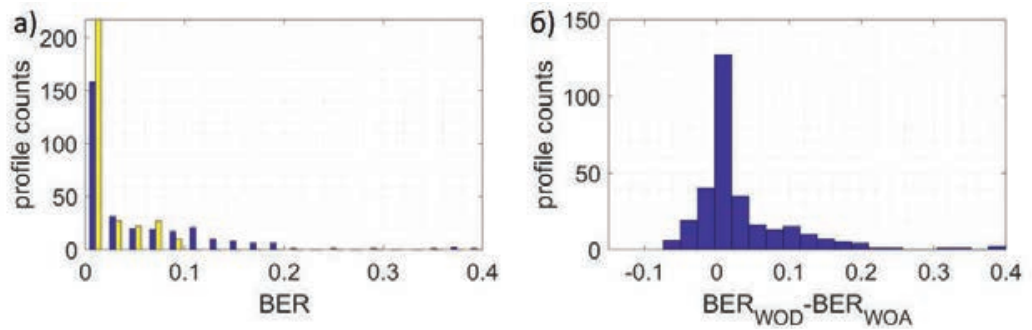

Рис. 3. а) гистограмма доли битовых ошибок декодирования для климатических (желтые столбики) и измеренных (синие столбики) профилей при фоновом АБГШ; б) гистограммаразности доли битовых ошибок декодирования для измеренных и климатических профилей при фоновом АБГШ.

Для измеренных профилей BER оказалась выше в среднем на 0.031 при стандартном отклонении 0.067. Такие отличия можно считать весьма существенными для приложений, требующих высокой точности декодирования, т.к. во многих случаях несколько процентов дополнительных ошибок могут означать невозможность устойчивой безошибочной передачи данных с приемлемой скоростью даже с помощью применения кодов, исправляющих ошибки [5].

Работа выполнена при поддержке государственного задания ИО РАН (тема 0149-2019-0011).

\section{Литература}

1. Rouseff D. Counterintuitive Results in Underwater Acoustic Communications. In: Zhou L., Xu W., Cheng Q., Zhao H. (eds) Underwater Acoustics and Ocean Dynamics. Singapore: Springer, 2016. 
2. Antonov J.I., Seidov D. et al. World Ocean Atlas 2009: Vol. 2: Salinity. S. Levitus / Ed. NOAA Atlas NESDIS 69. U.S. Government Printing Office. Washington: D.C., 2010. 184 p.

3. Boyer T.P., Antonov J.I. et al. World Ocean Database 2013. NOAA Atlas NESDIS 72. S. Levitus / Ed. A. Mishonov. Technical Ed. MD: Silver Spring, 2013. 209 p.

4. Porter M.B. The bellhop manual and user's guide: Preliminary draft. Heat, Light, and Sound Research Inc., La Jolla. CA, USA: Tech. Rep. 2011.

5. Proakis J. Digital Communications. NY USA: McGraw-Hill, 2001.

\title{
A.V. Shatravin \\ EVALUATION OF UNDERWATER ACOUSTIC COMMUNICATIONS PERFORMANCE BASED ON CLIMATOLOGICAL AND MEASURED SOUND SPEED PROFILES ON THE ARCTIC SHELF
}

\author{
Shirshov Institute of Oceanology, Russian Academy of Sciences, \\ Nakhimovskiy pr-kt, 36, Moscow, Russia, 117997, \\ Tel.:8(499)124-59-96,E-mail: ashatravin@ocean.ru
}

\begin{abstract}
We present resuls of numerical modeling of underwater acoustic communications based on climatological and measured vertical sound speed profiles on the shallow Arctic shelf of Russia. It is shown that the error level for measured profiles can be more than $6 \mathrm{~dB}$ higher than that for climatological profiles. Bit error rate for measured profiles is on average higher by 0.031 .
\end{abstract}

Keywords: underwater acoustic communications, Arctic shelf, climatological profiles, ray tracing

Acknowledgment: This work was supported by the state assignment of the IO RAS (topic 0149-2019-0011).

\section{References}

1. Rouseff D. Counterintuitive Results in Underwater Acoustic Communications. In: Zhou L., $\mathrm{Xu}$ W., Cheng Q., Zhao H. (eds) Underwater Acoustics and Ocean Dynamics. Springer, Singapore, 2016.

2. Antonov J.I. and Seidov D. et al. World Ocean Atlas 2009, Vol. 2: Salinity. S. Levitus, Ed.

NOAAAtlas NESDIS 69, U.S. Government Printing Office, Washington, D.C., 2010, 184 p.

3. Boyer T.P. and Antonov J.I. et al. World Ocean Database 2013, NOAA Atlas NESDIS 72,

S. Levitus, Ed., A. Mishonov, Technical Ed., Silver Spring, MD, 2013, 209 p.

4. Porter M.B. The bellhop manual and user's guide: Preliminary draft. Heat, Light, and Sound

Research, Inc., La Jolla, CA, USA: Tech. Rep., 2011.

5. Proakis J. Digital Communications. NY USA: McGraw-Hill, 2001. 